\title{
Bounds for the degree of polarization
}

\author{
J. W. Hovenier and C. V. M. van der Mee
}

Department of Physics and Astronomy, Free University, De Boelelaan 1081, 1081 HV Amsterdam, The Netherlands

Received July 24, 1995

Relations between the degree of polarization of the incident beam and that of the outgoing beam are discussed for a number of optical devices and for scattering by particles and surfaces. Rigorous upper and lower bounds for the degree of polarization of the outgoing beam are given for cases in which the degree of polarization of the incident beam is known. (C) 1995 Optical Society of America

A quantity of considerable interest in studies of polarized light is the degree of polarization of a beam of radiation. Every transformation of Stokes parameters described by a Mueller matrix may change the degree of polarization. The nature of this change, however, is not so obvious (see, e.g., Ref. 1).

The main purpose of this Letter is to present simple upper and lower bounds for the change of the degree of polarization of a beam that occurs on interaction with matter, as described by a Mueller matrix. Such bounds can be used for testing optical devices and numerical techniques.

We consider a quasi-monochromatic beam of radiation with Stokes parameters $I, Q, U$, and $V$ (see, e.g., Refs. 2 and 3). We write these Stokes parameters as elements of a column vector I, called the Stokes vector, and define the degree of polarization as

$$
p=\left(Q^{2}+U^{2}+V^{2}\right)^{1 / 2} / I .
$$

We always have $0 \leq p \leq 1$. If a beam of radiation with Stokes vector $\mathbf{I}_{1}$ and degree of polarization $p_{1}$ creates through linear processes a beam of radiation with Stokes vector $\mathbf{I}_{2}$ and degree of polarization $p_{2}$, we can write

$$
\mathbf{I}_{2}=\mathbf{M I}_{1}
$$

where the real $4 \times 4$ matrix $\mathbf{M}$ is called a Mueller matrix. Clearly, the corresponding change of the degree of polarization is, in general, a complicated function of the elements of $\mathbf{M}$ and $\mathbf{I}_{1}$. Instead of the elements $M_{i j}$ of the Mueller matrix, we will sometimes use

$$
m_{i j}=M_{i j} / M_{11} \text {. }
$$

Since $M_{11}$ is the largest element in absolute value, ${ }^{4,5}$ we have

$$
0 \leq\left|m_{i j}\right| \leq 1
$$

Similarly, we sometimes use the reduced Stokes parameters (i.e., the Stokes parameters divided by the first one) $q_{1}, u_{1}$, and $v_{1}$ for the incident beam.

A useful parameter turns out to be

$$
s=\left(m_{21}^{2}+m_{31}^{2}+m_{41}^{2}\right)^{1 / 2} .
$$

Apparently, $s$ represents the degree of polarization of a beam of outgoing radiation if the incident beam is unpolarized. Consequently, $s$ is the value of $p_{2}$ if $p_{1}=0$, and we have

$$
0 \leq s \leq 1 .
$$

In the following discussion we restrict ourselves to pure Mueller matrices, i.e., matrices that can be derived from $2 \times 2$ Jones matrices. This holds for optical devices such as quarter-wave plates and retarders and for scattering by one particle. Pure Mueller matrices have many interesting properties. ${ }^{5,6}$ For example, we can write

$$
I_{2}^{2}\left(1-p_{2}^{2}\right)=d^{2} I_{1}^{2}\left(1-p_{1}^{2}\right),
$$

where

$$
d^{2}=M_{11}^{2}\left(1-s^{2}\right) .
$$

Furthermore, we have

$$
s^{2}=m_{12}^{2}+m_{13}^{2}+m_{14}^{2} .
$$

Combining Eqs. (7) and (8) and using Eq. (2) gives

$$
1-p_{2}^{2}=\frac{M_{11}^{2}\left(1-s^{2}\right) I_{1}^{2}\left(1-p_{1}^{2}\right)}{\left(M_{11} I_{1}+M_{12} Q_{1}+M_{13} U_{1}+M_{14} V_{1}\right)^{2}},
$$

which may be written in the form [cf. Eq. (3)]

$$
\begin{aligned}
1-p_{2}^{2} & =\frac{\left(1-s^{2}\right)\left(1-p_{1}^{2}\right)}{\left(1+m_{12} q_{1}+m_{13} u_{1}+m_{14} v_{1}\right)^{2}} \\
& =\frac{\left(1-s^{2}\right)\left(1-p_{1}^{2}\right)}{\left(1+s p_{1} \cos \gamma\right)^{2}}
\end{aligned}
$$

where $\gamma$ is the angle between the vectors $\left\{m_{12}, m_{13}, m_{14}\right\}$ and $\left\{q_{1}, u_{1}, v_{1}\right\}$.

For given $s$ and $p_{1}$, the maximum $f\left(p_{1}, s\right)$ of $p_{2}$ will be assumed for $\gamma=0$, while the minimum $g\left(p_{1}, s\right)$ of $p_{2}$ will be assumed for $\gamma=\pi$. This gives

$$
\begin{aligned}
& f\left(p_{1}, s\right)=\frac{p_{1}+s}{1+s p_{1}}, \\
& g\left(p_{1}, s\right)=\frac{\left|p_{1}-s\right|}{1-s p_{1}} .
\end{aligned}
$$

We have thus found very simple upper and lower bounds for $p_{2}$. Note that $f\left(p_{1}, s\right)$ and $g\left(p_{1}, s\right)$ are both symmetric in $p_{1}$ and $s$. 


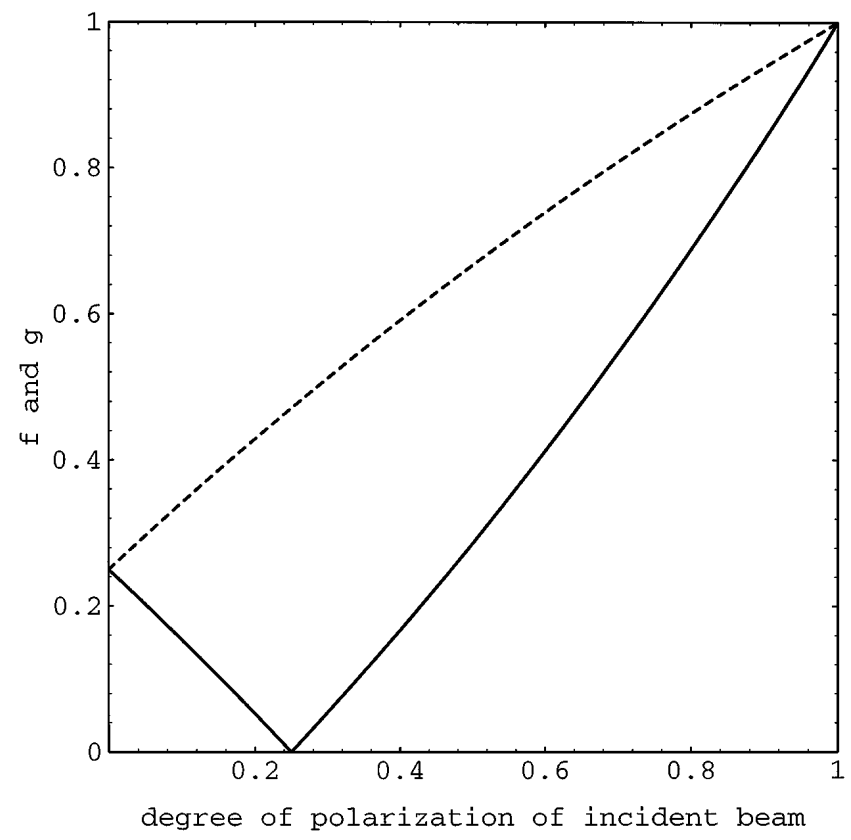

Fig. 1. Upper (dashed curve) and lower (solid curves) bounds for $p_{2}$ as functions of $p_{1}$. Here $s=1 / 4$.

Figure 1 shows $f\left(p_{1}, s\right)$ and $g\left(p_{1}, s\right)$ as functions of $p_{1}$ for $s=0.25$. If $0<s<1$ the bounds coincide for $p_{1}=0$ and $p_{1}=1$, yielding $p_{2}=s$ and $p_{2}=1$, respectively [cf. Eqs. (12) and (13)]. If $0<s<1$ the bounds for $p_{2}$ are reached if $\left\{q_{1}, u_{1}, v_{1}\right\}=\left( \pm p_{1} / s\right)\left\{m_{12}, m_{13}, m_{14}\right\}$, where the plus refers to the upper bound and the minus refers to the lower bound. If $0 \leq s<1$, the maximal difference $f-g$ is $2 s /\left(1+s^{2}\right)$, which occurs if $p_{1}=s$. If $s=0$ the bounds coincide for all values of $p_{1}$, and $p_{2}=p_{1}$. Similarly, the bounds coincide if $s=1$, and we then have $p_{2}=1$ for all values of $p_{1}$. This means that if a primary beam of unpolarized light creates completely polarized light through a pure Mueller matrix, the light is completely polarized for every state of polarization of the primary beam.

For practical purposes several conclusions may be drawn from Fig. 1 or Eqs. (11)-(13). We give three examples:

(1) If one has a pure Mueller matrix with $s=1 / 4$ it is not possible to obtain a beam with $p_{2} \geq 2 / 3$ when beams are used with $p_{1}<0.5$.

(2) If one wishes an instrument, characterized by a pure Mueller matrix with $0<s<1$, to depolarize as much as possible one should use light with $p_{1}=s$ and $\gamma=\pi$, i.e., $\left\{q_{1}, u_{1}, v_{1}\right\}=-\left\{m_{12}, m_{13}, m_{14}\right\}$, since then $p_{2}=0$.

(3) A pure Mueller matrix has the property that $p_{2}=1$ if $p_{1}=1$. For that reason a pure Mueller matrix is often called a totally polarizing Mueller matrix $^{7}$ or a nondepolarizing matrix. ${ }^{8}$ It should be realized, however, that a pure Mueller matrix may give light with $p_{2}<p_{1}$.

This concludes our discussion of pure Mueller matrices.

Let us now consider a Mueller matrix of the type

$$
\mathbf{M}=\left[\begin{array}{cccc}
a_{1} & b_{1} & 0 & 0 \\
b_{1} & a_{1} & 0 & 0 \\
0 & 0 & a_{3} & b_{2} \\
0 & 0 & -b_{2} & a_{3}
\end{array}\right],
$$

which holds, e.g., for the scattering matrix of an assembly of optically inactive homogeneous spheres, also called Mie scattering. In this case [cf. Eq. (2)]

$$
I_{2}^{2}\left(1-p_{2}^{2}\right)=\left(a_{1}^{2}-b_{1}^{2}\right)\left(I_{1}^{2}-Q_{1}^{2}\right)-\left(a_{3}^{2}+b_{2}^{2}\right)\left(U_{1}^{2}+V_{1}^{2}\right) .
$$

When we use Eq. (81) of Ref. 9,

$$
a_{3}^{2}+b_{2}^{2} \leq a_{1}^{2}-b_{1}^{2},
$$

Eq. (15) yields

$$
1-p_{2}^{2} \geq \frac{a_{1}^{2}\left(1-s^{2}\right) I_{1}^{2}\left(1-p_{1}^{2}\right)}{\left(a_{1} I_{1}+b_{1} Q_{1}\right)^{2}}
$$

which can be written as

$$
p_{2} \leq \frac{p_{1}+s}{1+p_{1} s}=f\left(p_{1}, s\right)
$$

Thus we find exactly the same upper bound as for a pure Mueller matrix.

Equation (15) also gives

$$
I_{2}^{2}\left(1-p_{2}^{2}\right) \leq\left(a_{1}^{2}-b_{1}^{2}\right)\left(I_{1}^{2}-Q_{1}^{2}\right),
$$

yielding

$$
1-p_{2}^{2} \leq \frac{\left(1-s^{2}\right)\left(1-q_{1}^{2}\right)}{\left(1-s\left|q_{1}\right|\right)^{2}}
$$

so that

$$
p_{2} \geq\left|\frac{s-\left|q_{1}\right|}{1-s\left|q_{1}\right|}\right| .
$$

Suppose we now have a Mueller matrix of the form

$$
\mathbf{M}=\operatorname{diag}\left(a_{1}, a_{2}, a_{3}, a_{4}\right) .
$$

Here

$$
p_{2}=\frac{\left(a_{2}^{2} q_{1}^{2}+a_{3}^{2} u_{1}^{2}+a_{4}^{2} v_{1}^{2}\right)^{1 / 2}}{a_{1}}
$$

which satisfies the inequalities

$$
\begin{aligned}
{\left[\frac{\min \left(\left|a_{2}\right|,\left|a_{3}\right|,\left|a_{4}\right|\right)}{a_{1}}\right] } & p_{1} \leq p_{2} \\
\leq & {\left[\frac{\max \left(\left|a_{2}\right|,\left|a_{3}\right|,\left|a_{4}\right|\right)}{a_{1}}\right] p_{1} . }
\end{aligned}
$$

In particular, since $\max \left(\left|a_{2}\right|,\left|a_{3}\right|,\left|a_{4}\right|\right) \leq a_{1},{ }^{9}$ we have

$$
p_{2} \leq p_{1} \text {. }
$$

Consequently, a diagonal Mueller matrix can never enhance the degree of polarization. 


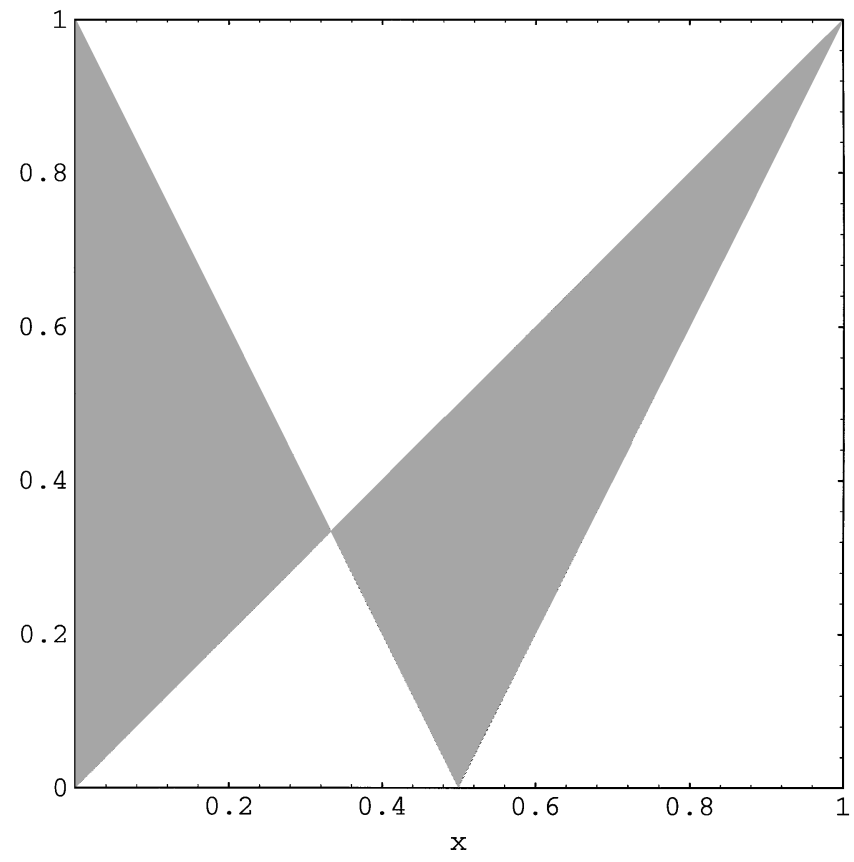

Fig. 2. Domains for $p_{2} / p_{1}$ (the shaded areas) for exact backscattering by the assemblies of particles described in the text.

An interesting special case is provided by exact backscattering by a small volume element comprising (i) randomly oriented particles having a plane of symmetry, such as ellipsoids, and/or (ii) particles and their mirror particles in equal numbers and in random orientation. The scattering matrix then is

$$
\mathbf{M}=\operatorname{diag}\left(a_{1}, a_{2},-a_{2}, a_{1}-2 a_{2}\right),
$$

where $a_{1} \geq a_{2} \geq 0 .{ }^{10}$ When we write $x=a_{2} / a_{1}$, inequalities (24) provide in this case

$$
\begin{aligned}
x p_{1} \leq p_{2} \leq(1-2 x) p_{1} & \text { if } x<1 / 3 \\
|1-2 x| p_{1} \leq p_{2} \leq x p_{1} & \text { if } x>1 / 3 \\
p_{2}=p_{1} / 3 & \text { if } x=1 / 3 .
\end{aligned}
$$

This is illustrated in Fig. 2.

It is a pleasure to thank J. F. de Haan and M. I. Mishchenko for their comments on an earlier version of this Letter.

\section{References}

1. Zhang-Fan Xing, J. Mod. Opt. 39, 461 (1992).

2. H. C. van de Hulst, Light Scattering by Small Particles (Wiley, New York, 1957; also Dover, New York, 1981).

3. J. W. Hovenier and C. V. M. van der Mee, Astron. Astrophys. 128, 1 (1983).

4. E. S. Fry and G. W. Kattawar, Appl. Opt. 20, 2811 (1981).

5. J. W. Hovenier, H. C. van de Hulst, and C. V. M. van der Mee, Astron. Astrophys. 157, 301 (1986).

6. J. W. Hovenier, Appl. Opt. 33, 8318 (1994).

7. R. Simon, Opt. Commun. 42, 293 (1982).

8. A. B. Kostinski, B. D. James, and W.-M. Boerner, J. Opt. Soc. Am. A 5, 58 (1988).

9. J. W. Hovenier and C. V. M. van der Mee, J. Math. Phys. 33, 3574 (1992).

10. M. I. Mishchenko and J. W. Hovenier, Opt. Lett. 20, 1356 (1995). 